\title{
FINITE-TO-ONE MAPS AND OPEN EXTENSIONS OF MAPS
}

\author{
J. K. KOHLI
}

\begin{abstract}
In the class of $p$-spaces it is shown that (a) infinite weight is not lowered by finite-to-one maps which are open except at finitely many points, (b) metrizability is inversely preserved under finiteto-one maps which are open except at finitely many points.
\end{abstract}

Proizvolov [6] showed that if the domain is a $p$-space, then weight ${ }^{1}$ is not lowered by open finite-to-one maps. In [3] Čoban showed that in the class of $p$-spaces, metrizability is inversely preserved under open finiteto-one maps. In [5] we obtained some improvements of these results. Proizvolov's theorem was extended to show that in the class of Čech complete spaces, weight is preserved by finite-to-one maps which are open except at finitely many points, and Čoban's result was extended to show that in the class of p-spaces, metrizability is inversely preserved under finiteto-one maps which are open except at finitely many points. Here we extend as follows these results of Proizvolov and Coban, essentially the same proof working for each of the respective generalizations.

Theorem. Let $f$ be a finite-to-one map of a p-space $X$ onto a p-space $Y$ such that $f$ is open except at finitely many points. Then weight $X \leq$ weight $Y$. If $Y$ is metrizable, so is $X$.

For the sake of completeness and to acquaint the reader with terminology and notation, we quote the following definition and results from [4].

Let $f$ be a function, not necessarily continuous, from a topological space $X$ into a topological space $Y$. We call a point $x \in X$ and its image $f(x) \in Y$ singular points of $X$ and $Y$, respectively, if there is an open set $U$ of $X$ containing $x$ whose image $f(U)$ is not a neighbourhood of $f(x)$. The function $f$ is open just in case there are no singular points of $X$ (or equivalently of $Y$ ).

Received by the editors October 16, 1972 and, in revised form, August 28, 1973.

AMS (MOS) subject classifications (1970). Primary 54C10, 54A25, 54E35; Secondary 54B 15.

Key words and phrases. p-space, feathering, weight, singular point, StoneČech compactification.

1 The author is thankful to the referee for pointing out that throughout the paper weight should stand for infinite weight. 
For each singular point $x$ of $X$, let $Y_{x}$ be a copy of $Y$. Let $W=X \oplus$ $\left(\bigoplus Y_{x}\right)$, where the second disjoint topological sum is taken over all singular points of $X$. By identifying each singular point $x \in X$ (with $X$ thought of as a subset of $W$ ) with its image $f(x)$ (as a point of $Y_{x} \subseteq W$ ), we arrive at a quotient space $X^{*}$ of $W$. The inclusion map $i_{x^{\circ}} X \rightarrow W$ composes with the quotient map $q: W \rightarrow X^{*}$ to give an embedding of $X$ into $X^{*}$. Hence $X^{*}$ may be considered as a superspace of $X$.

Let $f_{1}$ from $W$ onto $Y$ be the function whose restriction to $X$ is $f$ and whose restriction to each $Y_{x}$ is the identity map of $Y_{x}$ onto $Y$. Then the unique function $f^{*}: X^{*} \rightarrow Y$ satisfying $f^{*} \circ q=f_{1}$ is an open extension of f. If $f$ is continuous, then the function $f^{*}$ is continuous.

Throughout the paper, $S$ will denote the set of singular points of $X$, and the symbols $X^{*}, f^{*}, q$ will have the same meaning as in the above paragraphs. The set of natural numbers with discrete topology will be denoted by $N$; for any space $A, \beta A$ will denote its Stone-Čech compactification.

From here onward all the spaces are assumed to be completely regular and $T_{1}$, and all maps are assumed to be continuous onto. Therefore, $X^{*}$ is completely regular and $T_{1}$ ( see $[4,1.3]$ ).

Proof of Theorem. Since $f$ is finite-tooone, and since the set $S$ is finite, $f^{*}: X^{*} \rightarrow Y$ is an open finiteoto-one map. The Theorem follows immediately from the results mentioned in the beginning of this paper and the following lemma.

Lemma. If $X$ and $Y$ are p-spaces, and if the set $S$ is finite, then $X^{*}$ is a p-space.

Proof. First we show that $q(X)$ and each $q\left(Y_{x}\right)$ are $C$-embedded in $X^{*}$. Let $g$ be any continuous real-valued function on $q(X)$. Let $\tilde{g}$ be the extension of $g$ to $X^{*}$ which takes the constant value $g\left(q\left(x_{a}\right)\right)$ on $q\left(Y_{x_{a}}\right)$ for each $x_{a} \in S$. Then $\tilde{g}$ is continuous. Hence $q(X)$ is $C$-embedded in $X^{*}$. Now, let $h$ be any continuous real-valued function on $q\left(Y_{x}\right)$. Then $\tilde{h}=h \circ 1_{y} \circ f^{*}$, where $f^{*}$ is the open extension of $f$ and $1_{y}$ is the identity map of $Y$ onto $q\left(Y_{x}\right)$, is a continuous extension of $h$ to $X^{*}$.

Now, since $q(X)$ and $q\left(Y_{x}\right)$ are $C$-embedded in $X^{*}, \mathrm{cl}_{\beta X^{*}} q(X)=\beta q(X)$ and $\operatorname{cl}_{\beta X^{*}} q\left(Y_{x}\right)=\beta q\left(Y_{x}\right)$ for each $x \in S$. Hence $\beta X^{*}=\beta q(X) \cup\left(\cup \beta q\left(Y_{x}\right)\right)$, where the second union is taken over the set $S$.

Moreover, since the set $S$ is finite, $\beta X^{*}$ is obtained from $\beta W$ by identio fying each singular point $x \in X$ (with $X$ thought of as a subset of $\beta W$ ) with its image $f(x)$ (as a point of $Y_{x} \subseteq \beta W$ ).

In view of the above remarks and mathematical induction, it is enough 
to prove the Lemma when $S$ is a singleton, say, $S=\{x\}$. Thus, in this case $X^{*}=q(X) \cup q\left(Y_{x}\right)$ and, hence, $\beta X^{*}=\beta q(X) \cup \beta q\left(Y_{x}\right)$. Since $X$ and $Y$ are $p$-spaces [2], there exist featherings $\left\{\mu_{i}^{1}\right\}_{i \in N}$ and $\left\{\mu_{j}^{2}\right\}_{j \in N}$ of $q(X)$ and $q(Y)$ respectively. Let $(i, j) \in N \times N$ and let $U \in \mu_{i}^{1} \cup \mu_{j}^{2}$. If $q(x) \notin U$, let $\widetilde{U}=$ $U$. If $q(x) \in U \in \mu_{i}^{1}$, let $\tilde{U}=U \cup V$, where $q(x) \in V \in \mu_{j}^{2}$. If $q(x) \in U \in \mu_{j}^{2}$, let $\tilde{U}=U \cup W$, where $q(x) \in W \in \mu_{i}^{1}$. Let $\mu_{i j}=\left\{\tilde{U}: U \in \mu_{i}^{1} \cup \mu_{j}^{2}\right\}$. Then $\mu_{i j}$ is an open cover of $X^{*}$ in $\beta X^{*}$. Now we proceed to show that $\left\{\mu_{i j}\right\}_{(i, j) \in N \times N}$ is a feathering of $X^{*}$. Let $p \in X^{*}$ be any point. The following cases arise.

Case I. The set $q^{-1}(p)=\{x, f(x)\}$. Then

$$
\operatorname{St}\left(p, \mu_{i j}\right)=\operatorname{St}\left(p, \mu_{i}^{1}\right) \cup \operatorname{St}\left(p, \mu_{j}^{2}\right) \text {. }
$$

Therefore,

$$
\bigcap_{j=1}^{\infty} \mathrm{St}\left(p, \mu_{i j}\right)=\mathrm{St}\left(p, \mu_{i}^{1}\right) \cup \bigcap_{j=1}^{\infty} \mathrm{St}\left(p, \mu_{j}^{2}\right),
$$

and hence,

$$
\begin{aligned}
\bigcap_{i=1}^{\infty} \bigcap_{j=1}^{\infty} \mathrm{St}\left(p, \mu_{i j}\right)= & \left(\bigcap_{i=1}^{\infty} \mathrm{St}\left(p, \mu_{i}^{1}\right)\right) \\
& \cup\left(\bigcap_{j=1}^{\infty} \mathrm{St}\left(p, \mu_{j}^{2}\right)\right) \subseteq q(X) \cup q\left(Y_{x}\right)=X^{*} .
\end{aligned}
$$

Case II. The set $q^{-1}(p)=\left\{x_{a}\right\}$, where $x_{a} \in X$ and $x \neq x_{a}$. Then

$$
\mathrm{St}\left(p, \mu_{i j}\right) \subseteq \mathrm{St}\left(p, \mu_{i}^{1}\right) \cup \mathrm{St}\left(q(f(x)), \mu_{j}^{2}\right) \text {. }
$$

Therefore,

$$
\begin{aligned}
\bigcap_{i=1}^{\infty} \bigcap_{j=1}^{\infty} \operatorname{St}\left(p, \mu_{i j}\right) \subseteq & \left(\bigcap_{i=1}^{\infty} \operatorname{St}\left(p, \mu_{i}^{1}\right)\right) \\
& \cup\left(\bigcap_{j=1}^{\infty} \operatorname{St}\left(q(f(x)), \mu_{j}^{2}\right)\right) \subseteq q(X) \cup q\left(Y_{x}\right)=X^{*} .
\end{aligned}
$$

Case III. The set $q^{-1}(p)=\{y\}$, where $y \in Y_{x}$. Then

$$
\mathrm{St}\left(p, \mu_{i j}\right) \subseteq \mathrm{St}\left(p, \mu_{j}^{2}\right) \cup \mathrm{St}\left(q(x), \mu_{i}^{1}\right) .
$$

Therefore, 


$$
\begin{aligned}
\bigcap_{i=1}^{\infty} \bigcap_{j=1}^{\infty} \operatorname{St}\left(p, \mu_{i j}\right) \subseteq & \left(\bigcap_{j=1}^{\infty} \operatorname{St}\left(p, \mu_{j}^{2}\right)\right) \\
& \cdot\left(\bigcap_{i=1}^{\infty} \operatorname{St}\left(q(x), \mu_{i}^{1}\right)\right) \subseteq q(X) \cup q\left(Y_{x}\right)=X^{*}
\end{aligned}
$$

Thus $\left\{\mu_{i j}\right\}_{(i, j) \in N \times N}$ is a feathering of $X^{*}$. Hence $X^{*}$ is a $p$-space.

An altemative proof for the last part of the Theorem is given in [5]. In Example 3.5 of $[5], f$ is a on e-to-one map of a hereditarily paracompact Hausdorff nonmetrizable space onto a compact metric space which is open except at one point. Therefore the open extension $f^{*}: X^{*} \rightarrow Y$ is at most a two-toone map of a hereditarily paracompact Hausdorff nonmetrizable space on to a compact metric space. Thus the hypothesis on $X$ being a $p$-space cannot be re moved, either from the above Theorem or from the original theorems of Proizo volov and Čoban.

We do not know whether the inequality (weight $X \leq$ weight $Y$ ) in the above Theorem can be strengthened to equality. ${ }^{2}$ However, if, in addition, $Y$ is a Borel space (in particular, if $Y$ is a Čech complete space) or a subset of a perfectly normal compact space, then a theorem of Arhangel'skii [1] ensures the equality in the above theorem. Further, it may be observed that while the weight is not increased under open maps, it may be increased under maps which are open except at finitely many points. For, let $X=N, Y=N \cup\{p\}$, where $p \in \beta N-N$. Let $f$ be the map such that $f(1)=p$ and $f(n)=n-1$ for $n>1$. Then $f$ is a one-toone map of a metric space on to a hereditarily paracompact space which is open except at one point and weight $Y>$ weight $X$ $=K_{0}$.

Finally, the author wishes to thank the referee for many suggestions which have considerably improved the earlier presentation of the paper.

\section{REF ERENCES}

1. A. V. Arhangel'skir, Concerning the weight of topological spaces, Proc. Sympos. Topological General Topology and its Relations to Modern Anal. and Algebra, Academic Press, New York; Publ. House Czech. Acad. Sci., Prague, 1962, pp. 72-74. MR 30 \#4237.

2. - On a class of spaces containing all locally bicompact and all metric spaces, Mat. Sb. 67 (109) (1965), 55-85; English transl., Amer. Math. Soc. Transl. (2) 92 (1970), 1-39. MR 32 \#8299; $42 \# 3$.

3. M. M. Čoban, Open finite-to-one mappings, Dokl. Akad. Nauk SSSR 174 (1967), 41-48 = Soviet Math. Dokl. 8 ( 1967), 603-605. MR 35 \#6128.

${ }^{2}$ Added in proof. Equality is ensured by a result of Arhangel 'skii $[2,4.8]$. 
4. S. P. Franklin and J. K. Kohli, On open extensions of maps, Canad. J. Math. 22 (1970), 691-696. MR 41 \#9217.

5. J. K. Kohli, Open extensions of maps, finite-to-one maps and dimension (preprint).

6. V. V. Proizvolov, On finite-to-one open mappings, Dokl. Akad. Nauk SSSR 166 (1966), 38-40 = Soviet Math. Dokl. 7 (1966), 35-38. MR 32 \#6418.

HINDU COLLEGE, UNIVERSITY OF DELHI, DELHI-110007, INDIA 